\title{
Stability and Evolution of Supernova Fallback Disks
}

\section{Citation}

Menou, Kristen, Rosalba Perna, and Lars Hernquist. 2001. "Stability and Evolution of Supernova Fallback Disks." The Astrophysical Journal 559 (2): 1032-46. https://doi.org/10.1086/322418.

\section{Permanent link}

http://nrs.harvard.edu/urn-3:HUL.InstRepos:41381659

\section{Terms of Use}

This article was downloaded from Harvard University's DASH repository, and is made available under the terms and conditions applicable to Other Posted Material, as set forth at http:// nrs.harvard.edu/urn-3:HUL.InstRepos:dash.current.terms-of-use\#LAA

\section{Share Your Story}

The Harvard community has made this article openly available.

Please share how this access benefits you. Submit a story.

Accessibility 


\title{
Stability and Evolution of Supernova Fallback Disks
}

\author{
Kristen Menou, ${ }^{1}$ \\ Princeton University, Department of Astrophysical Sciences, Princeton NJ 08544, USA, \\ kristen@astro.princeton.edu \\ Rosalba Perna ${ }^{2}$ and Lars Hernquist \\ Harvard-Smithsonian Center for Astrophysics, 60 Garden Street, Cambridge MA 02138, USA, \\ rperna@cfa.harvard.edu, lars@cfa.harvard.edu
}

\begin{abstract}
We show that thin accretion disks made of Carbon or Oxygen are subject to the same thermal ionization instability as Hydrogen and Helium disks. We argue that the instability applies to disks of any metal content. The relevance of the instability to supernova fallback disks probably means that their power-law evolution breaks down when they first become neutral. We construct simple analytical models for the viscous evolution of fallback disks to show that it is possible for these disks to become neutral when they are still young (ages of a few $10^{3}$ to $10^{4}$ years), compact in size (a few $10^{9} \mathrm{~cm}$ to $10^{11} \mathrm{~cm}$ ) and generally accreting at sub-Eddington rates $(\dot{M} \sim$ a few $10^{14}-10^{18} \mathrm{~g} \mathrm{~s}^{-1}$ ). Based on recent results on the nature of viscosity in the disks of close binaries, we argue that this time may also correspond to the end of the disk activity period. Indeed, in the absence of a significant source of viscosity in the neutral phase, the entire disk will likely turn to dust and become passive. We discuss various applications of the evolutionary model, including anomalous X-ray pulsars and young radio pulsars. Our analysis indicates that metal-rich fallback disks around newly-born neutron stars and black holes become neutral generally inside the tidal truncation radius (Roche limit) for planets, at $\approx 10^{11} \mathrm{~cm}$. Consequently, the efficiency of the planetary formation process in this context will mostly depend on the ability of the resulting disk of rocks to spread via collisions beyond the Roche limit. It appears easier for the merger product of a doubly degenerate binary, whether it is a massive white dwarf or a neutron star, to harbor planets because its remnant disk has a rather large initial angular momentum, which allows it to spread beyond the Roche limit before becoming neutral. The early super-Eddington phase of accretion is a source of uncertainty for the disk evolution models presented here.
\end{abstract}

subject headings: X-ray: stars - accretion, accretion disks - supernovae: general - pulsars: general - stars: neutron - MHD - turbulence

\footnotetext{
${ }^{1}$ Chandra Fellow

${ }^{2}$ Harvard Junior Fellow
} 


\section{Introduction}

Fallback is a standard ingredient of contemporary core-collapse supernova scenarios. It occurs when the reverse shock, triggered by the impact of the ejected core material against the stellar envelope, reaches the central compact object (Colgate 1988; Chevalier 1989; see, e.g., Fryer \& Heger 2000 for recent core-collapse numerical simulations). Some of the fallback material will have a specific angular momentum in excess of the Keplerian value at the surface of the central compact object and is therefore expected to settle into a disk as it cools. The evolution of supernova fallback disks has rarely been investigated. Michel \& Dessler (1981, 1983) and Michel (1988) first pointed out the role that such a disk could play for the evolution of a young neutron star. Lin, Woosley \& Bodenheimer (1991) considered the evolution of a fallback disk in more detail, with a particular emphasis on the planetary formation process around young neutron stars. Recently, Chatterjee, Hernquist \& Narayan (2000) developed a model for Anomalous X-ray Pulsars (AXPs) based on the viscous evolution of a fallback disk and its magnetospheric interaction with the newly-formed neutron star. Alpar $(1999,2000)$ also presents an accretion scenario for AXPs. Marsden, Lingenfelter \& Rothschild (2001a; see also Marsden, Lingenfelter \& Rothschild 2001b) have recently considered the effect of fallback disks on the spin evolution of radio pulsars.

In this paper, we re-examine the evolution of supernova fallback disks from a general perspective. The viscous evolution of fallback disks should proceed according to the similarity solution derived by Cannizzo, Lee \& Goodman (1990; see also Pringle 1974; Lynden-Bell \& Pringle 1974) to study the accretion of the debris of a star that was tidally disrupted by a supermassive black hole. Numerical simulations (Cannizzo et al. 1990) show that after an initial, transient phase, the mass accretion rate drops off steadily as the supply of mass dwindles. The long term evolution of the accretion rate is then well approximated by a power-law decay with time. During their evolution, however, fallback disks enter a regime where they accrete at rates comparable to those at which disks in close binaries become subject to the thermal ionization instability ( $\sim 10^{16} \mathrm{~g} \mathrm{~s}^{-1}$ or so). The occurrence of the instability, which is at the origin of the large amplitude outbursts of Dwarf Novae and X-ray Transients (see reviews by Cannizzo 1993b; Osaki 1996; Lasota 2001), could signal the end of the power-law evolution of fallback disks.

We investigate this possibility here, and explore its consequences for the accretion scenario of AXPs and the planetary formation process around young compact objects. Meyer-Hofmeister (1992) and Mineshige, Nomoto \& Shigeyama (1993) were the first to discuss the role of the thermal ionization instability for supernova fallback disks. Their work was limited to the case where hydrogen is responsible for the instability and possibly leads to outbursts in the disk. The contribution in this paper is new in that we extend the relevance of the thermal ionization instability to metal-rich disks and we focus on the possibility that the instability could instead lead to the end of mass accretion in the disk. Note that, while this work was sparked by the recent applications of the fallback disk model described above, our results can be generally applied to isolated metal-rich disks formed by any mechanism. 
The outline of the paper is as follows. In $\S 2$, we show that metal-rich disks, such as supernova fallback disks, are subject to the well-known thermal ionization instability. In $\S 3$, we determine the typical characteristics of fallback disks at the time of the onset of the instability and argue that this could also be the end of the activity phase for the disk. In $\S 4$, we discuss applications of the scenario described in $\S 3$ to AXPs, the planetary formation process around newly-formed compact objects, doubly degenerate mergers, and other interesting cases. We comment on some of the limitations of our work in $\S 5$ before concluding in $\S 6$.

\section{Thermal Ionization Instability in Metal Disks}

\subsection{Method of Calculation}

The thermal-viscous stability of gaseous, thin accretion disks around compact objects has previously been investigated in many different contexts. The various existing results agree well with each other and show that Hydrogen-dominated disks are thermally and viscously unstable in regions of partial ionization corresponding to disk central temperatures $T_{c} \sim 10^{4} \mathrm{~K}$ (or, equivalently, disk effective temperatures $T_{\text {eff }} \lesssim 10^{4} \mathrm{~K}$; Meyer \& Meyer-Hofmeister 1981). Most of these studies considered disks of solar-composition material (see, e.g., Cannizzo 1993a; Ludwig, Meyer-Hofmeister \& Ritter 1994; Hameury et al. 1998 for recent calculations), as expected in close binaries where the mass accreted into the disk is supplied by a stellar companion. Smak (1983) was the first to study the stability of disks made of pure Helium, while Cannizzo (1984) presented a simplified time-dependent study of the evolution of such a Helium disk (see also Tsugawa \& Osaki 1997; El-Khoury \& Wickramasinghe 2000).

We focus here on disks made of metals. Because of the limited availability of pure metal opacities in the appropriate density and temperature ranges, we restrict our work to pure Carbon and pure Oxygen compositions. The case of solar composition material and pure Helium are also considered for comparison with previous results. The Rosseland-mean opacities were all taken from the OPAL database (Iglesias \& Rogers 1996) $)^{\text {f }}$. The opacities, at a specific mass density of $10^{-6} \mathrm{~g} \mathrm{~cm}^{-3}$, are shown as a function of temperature in Fig. 1, for the four compositions of interest: solar composition (solid line), pure Helium (short-dashed), pure Carbon (dotted) and pure Oxygen (long-dashed). In each case, the sudden opacity drop at temperatures $T \lesssim 10^{4} \mathrm{~K}$ corresponds to the recombination of the last free electron. This temperature differs from element to element because of different ground state ionization potentials. The plateau in the opacity curves at temperatures $T \gtrsim 10^{5} \mathrm{~K}$ corresponds to the electron scattering limit. We are mostly interested in low-temperature opacities for the present study.

The disk thermal equilibria are found by equating the local viscous dissipation rate $Q^{+}$to the

\footnotetext{
${ }^{3}$ The OPAL database website is http://www-phys.llnl.gov/Research/OPAL/
} 
radiative cooling rate $Q^{-}$, where $Q^{-}$is a function of the disk opacity. We neglect X-ray irradiation when calculating the thermal equilibria, but we consider its effects when discussing the evolution of supernova fallback disks in $§ 3$. The thermal equilibria are calculated from an extended grid of detailed models for the disk vertical structure, for different values of the surface density $\Sigma$ and the central temperature $T_{c}$, at a given radius $R$ from the central object of mass $M_{1}$ (see Hameury et al. 1998 for details on the numerical technique employed)f. Once a solution to the vertical structure (corresponding to a given $\Sigma, T_{c}$ and $R$ ) is known, the disk effective temperature $T_{\text {eff }}$ is uniquely determined. The equation of state adopted for the pure Helium, Carbon and Oxygen compositions is that of a perfect gas with a monoatomic adiabatic index $\gamma=5 / 3$. A detailed, tabulated equation of state is used for the solar composition case (see Hameury et al. 1998 for details).

\section{2. $\quad$ Results}

Figure 2 shows examples of thermal equilibrium curves (usually called "S-curves") in a surface density vs. effective temperature diagram. In each panel, the sections of the S-curves with negative slope are thermally and viscously unstable (see, e.g., Cannizzo 1993b for a review of the instability). Note that the only complete S-curves are those corresponding to the disk of solar composition, because this is the only case for which opacities in the neutral phase are available. In the solar composition case (having a detailed equation of state), we also show the typical effect on the S-curve when allowing for convection in the disk vertical structure (dotted line), in regions where the vertical temperature gradients are superadiabatic: the location of the unstable branch of the S-curve is somewhat affected. Since the exact role and magnitude of convection in thin accretion disks is not well understood, Fig. 2a basically shows that convection is a source of uncertainty for the derived stability criterion. The thermal equilibrium solutions are shown for different values of the viscosity parameter $\alpha$ to guarantee that opacities are available, for each composition, in the range of density and temperature covered by the model. The conclusions drawn below are essentially independent of the specific value of $\alpha$ adopted.

The presence of regions of negative slope in the curves of thermal equilibrium shown in Fig. 2 indicates that the corresponding disks are subject to the thermal ionization instability. The well-known results for a solar composition and a pure Helium disk are recovered here and the relevance of the instability is extended to metal-rich disks. Although this was only shown for pure Carbon and Oxygen compositions, we argue below that it is natural to generalize this result to disks of any composition.

The instability occurs at a characteristic effective temperature $T_{\text {eff,crit }}$ (where the slope in

\footnotetext{
${ }^{4}$ We also checked the validity of our results by solving the simplified vertically-averaged equation $Q^{+}=$ $(9 / 8) \nu \Sigma \Omega^{2}=Q^{-}=4 \sigma T_{c}^{4} /(3 \Sigma \kappa)$, valid for an optically-thick disk, where $\nu$ is the kinematic viscosity and $\kappa$ is the opacity.
} 
the S-curve changes from positive to negative), which is a function of the disk composition and the radius of interest. The dashed and solid lines in Fig. 2 (corresponding to $R=2 \times 10^{10}$ and $6 \times 10^{10} \mathrm{~cm}$, respectively) show that the variation of the equilibrium curves with radius is comparable for solar composition and metal-rich disks. On the other hand, the systematic offset of $T_{\text {eff,crit }}$ with composition can be related to the different ground state ionization potential in each case. Figure 2 of Mihalas et al. (1990), for example, shows that, for the densities relevant here and in order of decreasing temperature, Helium fully recombines first, followed by Oxygen, and then Hydrogen and Carbon with comparable values. This ordering is reflected in the temperatures at which the opacities drop at recombination in Fig. 1 and the ordering of $T_{\text {eff,crit }}$ for the various compositions shown in Fig. 2. 5 Other metals will have different ground state ionization potentials but there is every reason to expect that a disk of any composition will be subject to the same ionization instability.

\subsection{Global Stability Criterion}

The curves of thermal equilibrium can be used to derive a global stability criterion for the corresponding accretion disk. The relation $\dot{M} \propto T_{\text {eff }}^{4}$ between the disk accretion rate and its effective temperature implies that a disk becomes thermally unstable if it locally accretes at a rate below the value corresponding to $T_{\text {eff,crit }}(R)$. The well-known stability criterion for a solar composition disk is therefore (e.g. Hameury et al. 1998):

$$
\dot{M}_{\text {crit }}^{+}(R) \simeq 9.5 \times 10^{15} m_{1}^{-0.9} R_{10}^{2.68} \mathrm{~g} \mathrm{~s}^{-1},
$$

where $m_{1}$ is the mass of the central object in solar units and $R_{10}$ is the radius of interest in units of $10^{10} \mathrm{~cm}$. A disk which satisfies everywhere $\dot{M}(R)>\dot{M}_{\text {crit }}^{+}(R)$ is ionized and thermally (as well as viscously) stable. According to the results presented above, the stability criterion for a disk made of Helium or metals is quite similar, except for a different absolute scaling due to the eventual offset in the value of $T_{\text {eff,crit }}$, as shown in Fig. 2. For instance, the value of $T_{\text {eff,crit }}$ for a Helium disk being approximately twice that for a solar composition disk, the corresponding value of $\dot{M}_{\text {crit }}^{+}(R)$ should be multiplied by a factor of 8 or so. Since the deviation in Eq. (11) from the scaling $m_{1}^{-1} R^{3}$ followed by the local viscous dissipation rate (e.g. Frank, King \& Raine 1992) arises from small variations with radius of the disk density, and therefore opacity, at the temperature characteristic

\footnotetext{
${ }^{5}$ We checked that, whether the disk is made of solar composition material, pure Helium, Carbon or Oxygen, the value of $T_{\text {eff, crit }}$ depends only weakly on the viscosity parameter $\alpha$.

${ }^{6}$ Note that the relation between the disk central temperature (which determines the ionization state) and its effective temperature depends on the vertically integrated optical thickness of the disk. The ordering of $T_{\text {eff,crit }}$ therefore also depends on the gas opacity in each case.

${ }^{7}$ The viscous instability, which follows the exact same stability criterion, can generally be neglected because of its much longer growth time.
} 
of recombination, one may also expect slight deviations in the scaling of $\dot{M}_{\text {crit }}^{+}$with $m_{1}$ and $R$ for non Hydrogen-dominated compositions.

\section{Power-Law Evolution of a Supernova Fallback Disk}

In this section, we apply the results derived in $\S 2$ to the evolution of a supernova fallback disk. One may expect the self-similar evolution of this type of disk to break down when the thermal instability first sets in. This is because the evolution of the disk during the self-similar phase is regulated by viscosity, and when the disk recombines, the nature and magnitude of the viscosity will likely change (see $\S 3.3$ for further discussion on this issue). In the following, we estimate the time at which the thermal instability first sets in, and what the corresponding disk parameters are.

\subsection{Non-irradiated Disk}

In order to simplify the analysis below, we adopt the following global stability criterion to determine when a metal-rich disk becomes neutral:

$$
\dot{M}_{\text {crit }}^{+}(R) \approx \beta 10^{16} R_{10}^{3} \mathrm{~g} \mathrm{~s}^{-1} .
$$

This expression differs only slightly from that given in Eq. (11). We also neglect the very weak dependence on the viscosity parameter $\alpha$. Given that we ignore the exact composition of the disk (which determines the exact value of $T_{\text {eff, crit }}$ at which the disk becomes neutral), that we neglect the effects of convection on the disk vertical structure and that we are interested below in radii which are in the vicinity of $10^{10} \mathrm{~cm}$, the use of equation (2) is justified at an order-of-magnitude precision level or so. The factor $\beta$ in Eq. (2) captures all these uncertainties and any dependence on $m_{1} \neq 1$ (compare Eq. [1]).

In a steady (or quasi-steady) disk, the criterion is first met at the disk outer edge (where the gas in the disk becomes neutral first). Assuming that the disk evolves according to the self-similar solution of Cannizzo et al. (1990; for an electron scattering opacity), ${ }^{8}$ after an initial transient phase of duration $t_{0}$, the disk mass, $M_{d}$, outer radius, $R_{d}$, and mass accretion rate, $\dot{M}_{d}$, obey the relations:

$$
\begin{aligned}
M_{d}(t) & =M_{d}\left(t_{0}\right)\left(\frac{t}{t_{0}}\right)^{-3 / 16} \\
R_{d}(t) & =R_{d}\left(t_{0}\right)\left(\frac{t}{t_{0}}\right)^{3 / 8}
\end{aligned}
$$

\footnotetext{
${ }^{8}$ Cannizzo et al. found that the power-law evolution for a standard (Hydrogen-dominated) Kramers opacity is very similar to that for an electron scattering opacity. We use their analytical solution for the latter case here, which should be reasonably accurate even for metal-rich disks.
} 


$$
\dot{M}_{d}(t)=\dot{M}_{d}\left(t_{0}\right)\left(\frac{t}{t_{0}}\right)^{-19 / 16} .
$$

The equation for the disk radius results from the assumptions that most of the disk mass resides in its outermost regions and that the total angular momentum of the disk is conserved. One can easily check that most of the disk mass is indeed located at the outer edge in the power law solutions used here, and that little angular momentum is accreted with the gas that reaches the central compact object. The disk radius calculated in this way should approximate reasonably well the actual disk radius, in the limit of long time evolution and a large ratio of outer to inner disk radii (see Fig. 2 of Cannizzo et al. 1990 for a comparison of the results between a similarity solution and a detailed numerical integration).

The quantity

$$
\frac{\dot{M}_{d}(t)}{R_{d}^{3}(t)}=\frac{\dot{M}_{d}\left(t_{0}\right)}{R_{d}^{3}\left(t_{0}\right)}\left(\frac{t}{t_{0}}\right)^{-37 / 16}
$$

is a measure of the amount of viscous dissipation at the disk outer edge, as a function of time. According to Eq. (2), when

$$
\frac{\dot{M}_{d}(t)}{R_{d, 10}^{3}(t)} \approx \beta 10^{16} \mathrm{~g} \mathrm{~s}^{-1},
$$

the outermost annulus of the disk becomes neutral.

Let $M_{d}\left(t_{0}\right)$ and $R_{d}\left(t_{0}\right)$ be the initial mass and radius of the fallback material ending up in the disk. Various arguments concerning supernova explosions suggest that the amount of fallback is $<0.1 \mathrm{M}_{\odot}$ in the case of the formation of a neutron star (Lin, Woosley \& Bodenheimer 1991; Chevalier 1989), while conservation of angular momentum during the supernova explosion and the subsequent fallback would guarantee that the material brought back to the compact object by the reverse shock ends up at radii $\lesssim 10^{9} \mathrm{~cm}$ because of its initial location within the stellar core (of typical size a white dwarf radius). Recent numerical simulations of the pre-supernova evolution of rotating stars suggest values $j \approx 10^{16}-10^{17} \mathrm{~cm}^{2} \mathrm{~s}^{-1}$ for the specific angular momentum of the material surrounding the iron core just before collapse (Heger, Langer \& Woosley 2000). This corresponds to Keplerian radii $\approx 10^{6}-10^{8} \mathrm{~cm}$ around a solar mass central object. Below, when we specify the initial radius of a fallback disk, $R_{d}\left(t_{0}\right)$, it corresponds to choosing the mean specific angular momentum of the material forming the disk, or equivalently to choosing the total disk angular momentum $\propto M_{d}\left(t_{0}\right) R_{d}^{1 / 2}\left(t_{0}\right)$ for a given initial disk mass $M_{d}\left(t_{0}\right)$.

The fallback material with excess angular momentum, after cooling, will spread via a disk on a typical local viscous timescale, that we identify with the duration of the initial transient accretion phase (see Figure 3b of Cannizzo et al. 1990):

$$
t_{0} \equiv \frac{R^{2} \Omega_{K}}{\alpha c_{S}^{2}} \approx 6.6 \times 10^{-5}\left(T_{c, 6}\right)^{-1} R_{d, 8}^{1 / 2}\left(t_{0}\right) \mathrm{yrs},
$$

where $\Omega_{K}$ is the local Keplerian angular speed, $c_{S}$ is the sound speed, $R_{d, 8}\left(t_{0}\right)$ is the initial radius in units of $10^{8} \mathrm{~cm}$ and $T_{c, 6}$ is a typical temperature in the disk during this early phase, in units of 
$10^{6} \mathrm{~K}$. A viscosity parameter $\alpha=0.1$ and a solar mass central object were assumed when deriving Eq. (8). Note that $10^{6} \mathrm{~K}$ is a representative temperature for a disk annulus located at a radius $\sim 10^{8} \mathrm{~cm}$ from a stellar-mass central object accreting at the Eddington rate (see, e.g., Fig. 5 in Hameury et al. 1998 for typical temperatures). 9. This temperature explicitly depends on the radius of interest as well (in a non-trivial way), which is why we keep the scaling with $T_{c, 6}$ explicit in the above equation for $t_{0}$.

The accretion rate at the onset of the self-similar evolution phase is $\dot{M}_{d}\left(t_{0}\right) \sim M_{d}\left(t_{0}\right) / t_{0}$ (i.e. after the transient phase; see again Fig. 3b of Cannizzo et al. 1990), so that by combining Eqs. (35) and (7), we find that the outermost disk annulus becomes neutral after a time:

$$
t_{n} \approx 1.5 \times 10^{3} \operatorname{yrs}\left(\frac{M_{d}\left(t_{0}\right)}{\beta 10^{-3} \mathrm{M}_{\odot}}\right)^{16 / 37} T_{c, 6}^{-21 / 37} R_{d, 8}^{-75 / 74}\left(t_{0}\right) .
$$

This happens for a disk outer radius

$$
R_{d, 8}\left(t_{n}\right) \approx 570\left(\frac{M_{d}\left(t_{0}\right)}{\beta 10^{-3} \mathrm{M}_{\odot}}\right)^{6 / 37} T_{c, 6}^{6 / 37} R_{d, 8}^{16 / 37}\left(t_{0}\right),
$$

a disk mass

$$
M_{d}\left(t_{n}\right) \approx 4.2 \times 10^{-5} \mathrm{M}_{\odot} \beta^{3 / 37}\left(\frac{M_{d}\left(t_{0}\right)}{10^{-3} \mathrm{M}_{\odot}}\right)^{34 / 37} T_{c, 6}^{-3 / 37} R_{d, 8}^{21 / 74}\left(t_{0}\right),
$$

and a disk accretion rate

$$
\dot{M}_{d}\left(t_{n}\right) \approx 1.85 \times 10^{18} \mathrm{~g} \mathrm{~s}^{-1} \beta^{19 / 37}\left(\frac{M_{d}\left(t_{0}\right)}{10^{-3} \mathrm{M}_{\odot}}\right)^{18 / 37} T_{c, 6}^{18 / 37} R_{d, 8}^{48 / 37}\left(t_{0}\right) .
$$

For a low mass and low angular momentum disk $\left(M_{d}\left(t_{0}\right)=10^{-6} \mathrm{M}_{\odot}, R_{d, 8}\left(t_{0}\right)=0.01\right.$ and $\beta=1$ ), this gives:

$$
\begin{aligned}
t_{n} & \approx 7.5 \times 10^{3} \mathrm{yrs}\left(\times T_{c, 6}^{-21 / 37}\right) \\
R_{d}\left(t_{n}\right) & \approx 2.5 \times 10^{9} \mathrm{~cm}\left(\times T_{c, 6}^{6 / 37}\right) \\
\dot{M}_{d}\left(t_{n}\right) & \approx 10^{14} \mathrm{~g} \mathrm{~s}^{-1}\left(\times T_{c, 6}^{18 / 37}\right) .
\end{aligned}
$$

For a high mass and high angular momentum disk $\left(M_{d}\left(t_{0}\right)=10^{-2} \mathrm{M}_{\odot}, R_{d, 8}\left(t_{0}\right)=1\right.$ and $\beta=1$ ), this gives:

$$
\begin{aligned}
t_{n} & \approx 4 \times 10^{3} \mathrm{yrs}\left(\times T_{c, 6}^{-21 / 37}\right), \\
R_{d}\left(t_{n}\right) & \approx 8.3 \times 10^{10} \mathrm{~cm}\left(\times T_{c, 6}^{6 / 37}\right), \\
\dot{M}_{d}\left(t_{n}\right) & \approx 5.7 \times 10^{18} \mathrm{~g} \mathrm{~s}^{-1}\left(\times T_{c, 6}^{18 / 37}\right) .
\end{aligned}
$$

\footnotetext{
${ }^{9}$ Note that at super-Eddington accretion rates, the flow could in principle be much hotter than assumed here, reducing the initial viscous timescale $\left(t_{0}\right)$ accordingly. Uncertainties related to the super-Eddington accretion phase are further discussed in $\S 5$.
} 
A typical disk surface density is then:

$$
\Sigma \sim \frac{M_{d}\left(t_{n}\right)}{R_{d}^{2}\left(t_{n}\right)} \gg 1 \mathrm{~g} \mathrm{~cm}^{-2}
$$

so that the disk is still very optically thick to X-rays and its own radiation.

\section{2. $\quad$ Irradiated Disk}

The self-similar solutions to the viscous evolution of a thin accretion disk derived by Cannizzo et al. (1990) were obtained under the assumption of purely local viscous heating for the disk. This is clearly not appropriate for an irradiated disk, which is subject to an additional non-local heating. One can easily show, however, that even in the strong and steady irradiation limit (when the irradiation flux is time-independent and dominates over local viscous heating), the viscous evolution of the disk still obeys a similarity solution, with larger power law indices. Vrtilek et al. (1990; see also Cunningham 1976) showed that a vertically isothermal disk (satisfying hydrostatic equilibrium) has a vertical height $H$ which varies as a function of radius as $R^{9 / 7}$. The viscosity in this strongly irradiated $\alpha$-disk is then $\nu \equiv \alpha H^{2} \Omega_{K} \propto R^{15 / 14}$ (compare to $\nu \propto \Sigma^{2 / 3} R$ for the non-irradiated case; Cannizzo et al. 1990). Lynden-Bell \& Pringle (1974) have solved the general problem of the viscous evolution of a disk with kinematic viscosity $\nu \propto R^{n}$, for an arbitrary value of $n$. Using their similarity solutions (valid for $n \neq 2$ ), we find that a strongly and steadily irradiated $\alpha$-disk evolves viscously in a self-similar manner with a power law index $l=1 /(4-2 n)$ for the time evolution of the disk mass, so that $M_{d}(t) \propto t^{-7 / 13}$ and $\dot{M}_{d}(t) \propto t^{-20 / 13}$. This corresponds to a more rapid evolution than in the non-irradiated solution described by Eqs. (3-5). The strong-irradiation solution largely overestimates the strength of irradiation in that it assumes a constant flux hitting the disk. In a more realistic scenario, the viscosity $\nu$ will also be a decreasing function of time, as the accretion rate and the irradiation flux decrease with time.

The ionization stability criterion for an irradiated disk is also different because irradiation, as an extra source of heating, is able to keep the disk ionized where it would have been neutral otherwise. The stability of irradiated disks has previously been investigated in the context of accretion in low-mass X-ray binaries (see, e.g., Van Paradijs 1996; Dubus et al. 1999 and references therein). The intrinsic emission from the hot, young neutron star can be neglected for the evolution of a supernova fallback disk because it does not contribute more than $10^{33} \mathrm{ergs} \mathrm{s}^{-1}$. P To account for the effect of accretion-induced irradiation, we adopt the following global stability criterion:

$$
\dot{M}_{c r i t}^{i r r}(R) \approx \beta_{i r r} 10^{15} R_{10}^{2} \mathrm{~g} \mathrm{~s}^{-1}
$$

\footnotetext{
${ }^{10}$ Similarly, we neglect the extra source of heating due to radioactive decay because it only concerns the very early evolution of the supernova fallback material (see, e.g., Michel 1988).
} 
This expression differs only slightly from the formula given by Dubus et al. (1999): $\dot{M}_{c r i t}^{\text {irr }}(R) \simeq 1.5 \times 10^{15} m_{1}^{-0.4} R_{10}^{2.1} \mathrm{~g} \mathrm{~s}^{-1}$. Again, the use of Eq. (20) simplifies the derivation below and is justified at an order-of-magnitude precision level or so. A specific calibration for the disk geometry and albedo in low mass X-ray binaries (see Dubus et al. 1999) was used to derive the absolute scaling in Eq. (20). Consequently, the factor $\beta_{i r r}$ in Eq. (20) covers uncertainties concerning the disk composition, geometry and albedo, as well as any dependence on $m_{1} \neq 1$.

A comparison between Eq. (2) and Eq. (20) suggests that disk irradiation dominates the stability properties of disks which extend beyond $\sim 10^{9} \mathrm{~cm}$. The solutions derived in $\S 3.1$ are therefore valid only for disk initial parameters such that the disk becomes neutral before it reaches radii $\sim 10^{9} \mathrm{~cm}$ or so. If irradiation becomes important during the disk evolution, the quantity $\dot{M}_{d} / R_{d}^{2}$, which measures the local heating due to disk irradiation, must this time be used to determine the disk stability properties. As a fallback disk spreads, it may reach radii $\sim 10^{9} \mathrm{~cm}$, where irradiation starts becoming important for its energy budget. The disk evolution accelerates slowly as the disk spreads further and irradiation becomes more and more important (driving the disk more and more toward the isothermal case). The exact solution to this problem can only be obtained numerically (especially because the viscosity is then a function of time).

In Appendix A, we consider two simple cases for the evolution of an irradiated disk. In one case, labeled "weakly-irradiated disk," we assume that the power-law evolution of the disk remains as slow as in the non-irradiated case, while we determine the stability properties of the disk including the effect of irradiation (Eq. [20]). In the other case, labeled "strongly-irradiated disk," we assume that as soon as the disk reaches $10^{9} \mathrm{~cm}$ and irradiation becomes significant, its evolution becomes more rapid and follows the similarity solution derived above for a vertically isothermal disk. The main result obtained from these models is that, even when the effects of irradiation are taken into account, a fallback disk is expected to become neutral when it is still young, compact and accreting at substantially sub-Eddington rates (see Appendix A). We emphasize that the "weakly-irradiated" case is probably a better approximation than the "strongly-irradiated" one because of the extreme assumptions made concerning the strength of irradiation in the latter case (Dubus et al. 1999, for instance, note that even the irradiated disks in low mass X-ray binaries are far from being vertically isothermal). In Appendix B, we also show that a relatively compact disk is a general feature of the models that remains valid even for an arbitrarily fast power law evolution of the fallback disk.

\subsection{Subsequent Evolution}

The immediate outcome of the thermal ionization instability, when it is first triggered in the outermost regions of the ionized disks of close binaries, has been studied numerically in detail and is relatively well understood. It generally leads to the outside-in propagation of a cooling (or equivalently recombination) front that reaches the central compact object relatively quickly because the instability is successively triggered in disk annuli at smaller and smaller radii. The 
front propagation typically takes only days to tens of days, depending on the role of irradiation at controlling its propagation (see, e.g., Cannizzo 1993a; Menou, Hameury \& Stehle 1999; Dubus, Hameury \& Lasota 2001). Because of the strong analogy between fallback disks and disks in X-ray Transients (in terms of size and susceptibility to the same ionization instability), there is every reason to believe that this phase should proceed identically in a fallback disk. Right after the front propagation, the surface density profile in the disk is approximately $\Sigma \propto R$, because of the important redistribution of mass and angular momentum driven by the front during its propagation (putting most of the mass in the outer disk; see, e.g., Cannizzo 1993a; Hameury et al. 1998; Dubus et al. 2001 for numerical examples).

The subsequent disk evolution, after it becomes entirely neutral, is less certain. It mostly depends on the nature and magnitude of viscosity in a neutral disk, which is not well understood. There are many reasons to believe that the MHD turbulence resulting from the magneto-rotational instability (Balbus \& Hawley 1991; 1998; Hawley, Gammie \& Balbus 1996) cannot be self-sustained in the neutral disks of X-ray Transients and Dwarf Novae (i.e. during quiescence) because they are too weakly ionized (Gammie \& Menou 1998; Fleming, Stone \& Hawley 2000; Menou 2000). $\square$ Convection does not provide the necessary outward angular momentum transport for accretion to proceed (Ryu \& Goodman 1992; Stone \& Balbus 1996; Cabot 1996), while the possibility of hydrodynamical turbulence is not supported by the same simulations of Keplerian disks which show the development of the Balbus-Hawley instability in the magnetized case (Hawley, Balbus \& Winters 1999). Purely theoretical arguments have also been presented against hydrodynamical turbulence (Balbus \& Hawley 1998; Hawley, Balbus \& Winters 1999; but see Richard \& Zahn 1999 for a different view). This leads to the interesting possibility that angular momentum transport could be associated with the tidal influence of the companion star in the neutral disks of close binaries (Spruit 1987), in which case isolated disks like the fallback disks considered here could be essentially passive when they become neutral (Menou 2000). [2]

We note that the results concerning MHD turbulence in neutral disks established for Dwarf Novae and X-ray Transients cannot be directly applied to fallback disks because of their different composition.[13 Indeed, the presence of MHD turbulence mainly depends on the ionization fraction in the disk, which is essentially an exponential function of the disk central temperature (via the Saha equation in LTE; Gammie \& Menou 1998). The central temperature itself depends on the opacity in the neutral/molecular phase $\left(T_{c} \propto \tau^{1 / 4}\right.$ in an optically thick disk at a given $T_{\text {eff }}$; Frank

\footnotetext{
${ }^{11}$ Note that Hall effects, which are small in the present context but have a potentially destabilizing effect, have not been included in these studies (Balbus \& Terquem 2001).

${ }^{12}$ Another mechanism that has been proposed to transport angular momentum in disks, the tidally-induced parametric instability, also relies on the presence of a companion star (see Balbus \& Hawley 1998; Goodman 1993).

${ }^{13}$ In their study of disks around supermassive black holes, Menou \& Quataert (2001) pointed out that the disk size also matters for the quality of gas to magnetic coupling in the neutral phase resulting from the thermal ionization instability. In that respect, fallback disks are comparable to disks in dwarf novae and X-ray transients.
} 
et al. 1992), so that the properties of magnetic coupling of the gas in a metal disk could potentially be different from those in a solar composition disk.

The opacity of a neutral metal-rich disk will be determined by a complex chemistry (such as, for instance, the formation of $\mathrm{CO}$ molecules in a metal-rich gas) which depends on the actual composition and is presently difficult to characterize. We note, however, that Lin et al. (1991) advocate a mixture of $\mathrm{Fe}, \mathrm{Si}, \mathrm{O}, \mathrm{He}$ and perhaps traces of $\mathrm{H}$ in their discussion of supernova fallback disks (around a neutron star). This is not a mixture that would allow the formation of all the molecules whose contribution to the opacity is important in a solar composition gas (in particular those based on carbon and hydrogen chemistry; e.g. Alexander \& Ferguson 1994). This shows that smaller opacities are actually quite possible in a fallback disk (as compared to a solar composition disk) in the neutral/molecular phase. If the fallback disk cools even further, the dust grains which start forming would contribute much more to the disk opacity (per unit mass) than in a solar composition gas because the disk is made entirely of metals. But this time also corresponds to the end of the gaseous phase for the disk as it presumably turns entirely to dust (contrary to a solar composition disk) and becomes unlikely to sustain MHD turbulence. Consequently, if the neutral/molecular opacities in fallback disks are indeed not substantially larger than in the solar composition case, a passive gaseous disk, soon to turn to dust, appears as the plausible outcome of the evolution outlined in $\S 3.1$ and $\S 3.2$, given our current understanding of the nature of viscosity

in the disks of transient close binaries. The time the disk first becomes neutral will then signal the end of its activity period.

We adopt this view in what follows, although we comment on the consequences of an eventual residual viscosity for neutral fallback disks in $\S 5$. We note that, even for passive neutral disks, there could in principle still be some residual disk accretion in surface layers which are ionized by the X-rays coming from the central, hot neutron star (in a manner similar to that envisioned for T-Tauri disks by Gammie 1996), if the disk geometry allows it. This possibility will probably not exist in the case of accretion onto a central black hole (weaker self-irradiation).

\section{Applications}

\subsection{Anomalous X-ray Pulsars}

\subsubsection{General Results}

The evolutionary scenario described in $§ 3$ has a direct application as an accretion model for Anomalous X-ray Pulsars (AXPs), superseding that developed by Chatterjee et al. (2000). In this model, the evolution of a newly-born neutron star mainly depends on the nature of its magnetospheric interaction with the surrounding fallback disk. Chatterjee et al. find that, depending on the initial values of the neutron star spin period, magnetic field strength and disk mass, a system can evolve into an X-ray luminous AXP, where the neutron star has been 
substantially slowed down by the torque exerted by the disk during a long propeller phase, or into a radio pulsar which did not interact as strongly with its surrounding disk (located beyond the pulsar light cylinder at late times). Chatterjee \& Hernquist (2000) showed that this scenario can reproduce the inferred distribution of AXP spin periods, luminosities and age distributions for reasonable and broad distributions of the model input parameters.

One of the shortcomings of the Chatterjee et al. model for AXPs is the necessity to postulate that the end of the activity period for the fallback disk (required to explain the inferred ages of AXPs) corresponds to a transition to a radiatively inefficient Advection-Dominated Accretion Flow (ADAF; see Narayan, Mahadevan \& Quataert 1998 for a review). The AXP model also requires the onset of a propeller effect in this late evolutionary phase to effectively reduce the accretion rate onto the central neutron star.

These requirements are no longer needed if the viscous evolution scenario described in $\S 3$ is adopted. In our model, the disk always remains geometrically thin, and the end of the disk activity period (and therefore of the AXP activity) simply corresponds to the time when the disk suddenly becomes neutral and passive under the action of the thermal ionization instability. This model, using disk accretion physics tested in close binary systems and no fine tuning of parameters (except a choice of initial disk mass and angular momentum), can provide a satisfying explanation for the inferred ages and luminosities of AXPs. At the same time, this fallback disk scenario can accommodate the constraints on disk sizes around AXPs because the end of the activity period corresponds to such an early time, that the disk did not have time to spread viscously very much. We have shown that, if the fallback material ending up in the disk has low initial mass and angular momentum, the model can account for disk sizes as small as a few $10^{9} \mathrm{~cm}$. For example, a specific non-irradiated fallback disk with $M_{d}\left(t_{0}\right)=10^{-4} \mathrm{M}_{\odot}, R_{d, 8}\left(t_{0}\right)=0.03$ and $\beta=1$ becomes neutral when:

$$
\begin{aligned}
t_{n} & \approx 2 \times 10^{4} \mathrm{yrs}\left(\times T_{c, 6}^{-21 / 37}\right), \\
R_{d}\left(t_{n}\right) & \approx 8.6 \times 10^{9} \mathrm{~cm}\left(\times T_{c, 6}^{6 / 37}\right), \\
\dot{M}_{d}\left(t_{n}\right) & \approx 6.4 \times 10^{15} \mathrm{~g} \mathrm{~s}^{-1}\left(\times T_{c, 6}^{18 / 37}\right),
\end{aligned}
$$

in good agreement with the inferred properties for AXPs. We further discuss the issue of disk size in $\S 4.1 .2$, together with that of the strength of disk irradiation in AXPs. By virtue of the power law evolution in time, the model predicts that most systems should be observed relatively close to the end of their activity period. The fact that the systems are necessarily observed before $t_{n}$, i.e. before the disk has reached its final radial extent, only helps to explain the compact sizes inferred. In our view, the simplicity of this model, the classic nature of its ingredients, and its ability to reproduce reasonably well the observed characteristics of AXPs without parameter fine-tuning make it a strong alternative to the magnetar model (Thompson \& Duncan 1996; Heyl \& Hernquist 1997).

We note a possible difficulty for the proposed AXP model to explain disk sizes much smaller than inferred to date. The magnetospheric truncation radius of the disk is 
$R_{m} \approx 2.5 \times 10^{8} \mathrm{~cm} B_{12}^{4 / 7} \dot{M}_{16}^{-2 / 7}$, where $B_{12}$ is the neutron star surface magnetic field strength in units of $10^{12} \mathrm{G}$ and $\dot{M}_{16}$ the accretion rate is units of $10^{16} \mathrm{~g} \mathrm{~s}^{-1}$ (e.g. Frank et al. 1992). Clearly, the disk outer radius cannot be much smaller than $R_{m}$. We also note that the disk must acquire some angular momentum as it spins down the neutron star. This gain is moderate in the quasi-equilibrium "tracking-phase" model of Chatterjee et al. (2000; see discussion in §5) but its effect is to increase the disk size compared to the predictions made in $\S 3$. These complications show that a detailed numerical integration is probably required to obtain more reliable estimates of the disk characteristics when it becomes neutral, especially in those cases where the initial disk mass and angular momentum are small enough that the disk outer radius, $R_{\text {out }}$, is not much larger than the inner edge, $R_{\text {in }}=R_{m}$.

\subsubsection{Disk Size and Irradiation}

Upper limits to the optical flux from AXPs provide useful constraints on all accretion models of these systems (see, e.g., Hulleman et al. 2000a, Perna, Hernquist \& Narayan 2000; Perna \& Hernquist 2000 and references therein). The tightest existing constraint is that given by the recent identification of a possible optical counterpart to the AXP 4U 0142+61 (Hulleman, van Kerkwijk \& Kulkarni 2000b). Hulleman et al. (2000b) infer that only a compact accretion disk, of outer radius $R_{\text {out }} \lesssim 0.05 R_{\odot} \approx 3.5 \times 10^{9} \mathrm{~cm}$ (with uncertainties in the source distance and the strength of irradiation), is allowed in $4 \mathrm{U} 0142+61$. By analogy, one can expect comparable constraints on the maximal radial extent of hypothetical disks around other AXPs.

The evolutionary model for supernova fallback disks described in $\S 3$ appears consistent with these restrictions: active fallback disks should be compact because they do not have time to spread extensively before they become neutral (and passive), when the thermal ionization instability first sets in. We have shown that, with a choice of rather small initial mass and angular momentum for the disk, a disk size of a few $\sim 10^{9} \mathrm{~cm}$ may be expected at the end of the activity phase. ${ }^{\mathbb{4}}$ Although this value is only an estimate of the disk radius and the models are subject to various uncertainties (see $\S 5$ for a discussion), the possibility of explaining very compact disks with a reasonable choice of initial disk parameters is quite satisfying given the level of simplicity of the model.

¿From the optical constraints available so far, and the very high ratio $F_{X} / F_{\text {opt }} \gtrsim 10^{4}$ (Hulleman et al. 2000b) measured for the AXP $4 \mathrm{U} 0142+61$, it is clear that, if there is a disk in

\footnotetext{
${ }^{14}$ The disk sizes considered here are smaller than in Hulleman et al. (2000a), Perna, Hernquist \& Narayan (2000), and Perna \& Hernquist (2000). We attribute this difference partly to smaller disk mass and angular momentum and partly to a different description of the initial super-Eddington accretion phase. While Perna et al. use the self-similar solution of Cannizzo et al. (1990) in this regime for which it does not strictly apply, we assume here that the early evolution occurs on a relatively long viscous timescale associated with efficient cooling of the disk. None of these two possibilities is fully satisfying but there is presently no robust solution to this problem (see $\S 5$ ).
} 
this source, it must be of much smaller size and much less irradiated than typical disks in low mass X-ray binaries. The weakness of irradiation simply results from the small disk size. For instance, at a typical AXP luminosity of a few $10^{35} \mathrm{erg} \mathrm{s}^{-1}$, Hulleman et al. (2000a) show that the R band flux is typically dominated by viscous dissipation for disk radii $\lesssim$ a few $10^{9}$ to $10^{10} \mathrm{~cm}$. The weak irradiation also means that the evolution model for a non-irradiated disk described in $\S 3.1$ is more appropriate than the models with irradiation described in Appendix A.

It is also worth mentioning that a careful comparison between the light expected from a supernova fallback disk and the observational constraints probably requires a model more elaborate than a simple Shakura-Sunyaev $\alpha$-disk. Although it is true that the emission from a disk evolving viscously as considered in $\S 3$ is similar to that of an $\alpha$-disk for the bulk of the disk, it is no longer true close to the disk outer edge. There, the disk surface density, and the viscous dissipation proportional to it, fall with radius faster than in an $\alpha$-disk (see, e.g., Eq. [13] in Cannizzo et al. 1990). Given that most of the disk optical light (e.g. R band; Hulleman et al. 2000a) comes from the outer regions for the typical disk sizes considered, inferring an accurate limit on the disk radius from the observations probably requires that this effect be taken into account. We acknowledge that the disk radii as estimated in this paper do not take this effect into account either.

\subsubsection{Observational Signatures}

If supernova fallback disks power AXPs, these systems may reveal signatures of the presence of a disk (most likely in optical). The best analogy would then be to disks in low mass X-ray binaries if fallback disks are strongly irradiated, but the likelihood that they are not significantly irradiated because of their small sizes make disks in Cataclysmic Variables (CVs; see Warner 1995 for a review) perhaps the best equivalent. A characteristic signature of these disk is a broad, double-peaked emission line (function of orientation), although the line may not be that broad if $R_{\text {in }}=R_{m}$ is not much smaller than $R_{\text {out }}$ for fallback disks (see the analogy with intermediate polars in Warner 1995). Many of the atomic transitions available to disks of solar composition may not be available to fallback disks, however, because of their high metallicity. It is still possible that (presumably weak) hydrogen lines could be observed if the disk contains a small fraction of hydrogen. In X-rays, the possibly high iron content of the (cold) disk may favor energy emission through the iron K $\alpha$ fluorescence line (see, e.g., George \& Fabian 1991), although a sufficient flux of hard X-rays reprocessed by the disk may not be available to power the line if supernova fallback disks are not significantly irradiated.

The variability properties of fallback disks may be quite different from those of disks in CVs as well. The two variability phenomena whose origin has been identified in CVs (flickering and dips) do not apply to fallback disks because they do not possess a bright spot, where the stream of mass transferred from the companion impacts the disk in close binaries. Warner (1995) notes, however, that part of the flickering seen in CVs is apparently intrinsic to the disk, so that some flickering from supernova fallback disks may be expected. 
If the optical light from AXPs were dominated by X-ray reprocessing, it would follow any $\mathrm{X}$-ray fluctuation, after a delay corresponding to the light travel time over the disk radial extent. Optical pulsations could potentially be observed despite the strong isotropization of the X-ray emission due to general relativistic effects in the vicinity of the neutron star (Perna \& Hernquist 2000). In the case of the AXPs, however, because of the very small size inferred for the disk, the optical emission is likely dominated by viscous dissipation, so that no optical pulsations would be expected. In the magnetar interpretation for the AXPs, no detailed predictions exist for the mechanism of optical emission and its characteristics. However, if this emission is due to magnetospheric effects as in isolated pulsars then, most likely, it will be highly pulsed as observed, for example, in the case of the Crab pulsar (Warner et al. 1969).

\subsection{Radio Pulsars}

The model of Chatterjee et al. (2000) for the magnetospheric interaction of a young neutron star with a surrounding fallback disk predicts that many systems will not be X-ray luminous sources but instead become standard radio pulsars. This happens when the disk magnetospheric truncation occurs beyond the light cylinder in the system. Chatterjee \& Hernquist (2000) estimate that, for reasonable distributions of input model parameters, $\sim 80 \%$ of newly born neutron stars will follow the radio pulsar evolutionary path.

The evolution of a fallback disk in this context will first depend on its capacity to survive in the environment of an energetic pulsar wind. If the disk survives, then it seems plausible that it will also evolve according to the scenario described in $\S 3$. The structure of the magnetic field outside the light cylinder, $R_{\mathrm{lc}}$, is quite uncertain, but it is clear that if the disk penetrates inside $R_{\mathrm{lc}}$, it will encounter a strong centrifugal barrier there because the magnetosphere rotates at a much faster rate than the local Keplerian value. Ejection via the propeller effect would probably follow (Illarionov \& Sunyaev 1975), allowing the disk to lose mass at its inner edge (as required for the model in $\S 3$ to apply).

If so, the neutron star will also be spun down by the propeller effect. Determining the total amount of spin-down applied during the disk activity period requires a detailed accretion+torque model that will not be investigated here. We note, however, that in the magnetospheric scenario of Chatterjee et al. (2000), radio pulsars are the results of the evolution of the young neutron stars with the least massive disks. Over their activity period, which is of shorter duration than for more massive disks, these disks will therefore apply comparatively lower integrated spin down torques. Order-of-magnitude estimates suggest that the integrated torque applied by a low mass and low angular momentum disk such as those considered in $\S 3$ would result in a period increase of at most a factor of a few for a radio pulsar initially spinning at $15 \mathrm{~ms}$. It appears therefore possible to keep fast spinning radio pulsars in this scenario, even if they used to accrete from a gaseous fallback disk early on. Another possibility, of course, is that the effect of the disk on the neutron star is negligible because it is rapidly disrupted by the pulsar wind. An important difference between 
these two evolutionary scenarios for radio pulsars is that, in the case of disk survival, a remnant disk of rocks is also expected which could later lead to planet formation, as explained below. We note that, along those lines, Marsden, Lingenfelter \& Rothschild (2001a; see also Marsden, Lingenfelter \& Rothschild 2001b) have recently proposed that torques due to a fallback disk could contribute to spinning down radio pulsars faster than anticipated from magnetic dipole radiation alone.

\subsection{Planet Formation}

The cold and metal-rich disk resulting from the evolutionary scenario outlined in $§ 3$ appears to be an ideal site for the formation of dust, planetesimals and perhaps planets. The notion that a supernova fallback disk spreads viscously until it reaches conditions appropriate to the formation of planets was previously investigated by Lin et al. (1991). One of the proposed scenarios for the origin of the planets around the radio pulsar PSR 1257+12 (Wolszczan \& Frail 1992; Wolszczan 1994) invokes this same type of fallback disk evolution (see Phinney \& Hansen 1993 for a review).

The fact that the disk may become neutral and perhaps inviscid when the thermal ionization instability first sets in affects the planetary formation scenario dramatically. This is because the disk may not have time to spread to large enough radii for planets to form. For the specific initial disk parameters discussed in $\S 3$, the disk outer radius is $<10^{11} \mathrm{~cm}$ when it becomes neutral, even for the large values of the disk initial parameters $R_{d}\left(t_{0}\right)=10^{8} \mathrm{~cm}$ (corresponding to a specific angular momentum $j=10^{17} \mathrm{~cm}^{2} \mathrm{~s}^{-1}$ around a solar mass object) and $M_{d}\left(t_{0}\right)=10^{-2} M_{\odot}$. Although the "strongly-irradiated" disk model described in Appendix A predicts larger final radii, we argued that it likely overestimates the real effect of irradiation on the disk evolution.

The tidal disruption radius for a self-gravitating body of density $\rho \approx 1 \mathrm{~g} \mathrm{~cm}^{-3}$ around a neutron star of mass $M_{1}=1.4 M_{\odot}$ is (e.g. Aggarwal \& Oberbeck 1974)

$$
R_{\mathrm{tid}} \approx\left(\frac{M_{1}}{\rho}\right)^{1 / 3} \approx 10^{11} \mathrm{~cm}
$$

This shows that, according to our models, a fallback disk will not in general spread enough during its gaseous phase to allow immediate planet formation. Hansen (2001; see also Phinney \& Hansen 1993) recently investigated the viscous evolution of a disk in PSR $1257+12$ in scenarios where the disk becomes inviscid below a temperature of $3000 \mathrm{~K}$. He considers scenarios which allow more total angular momentum for the disk than the fallback scenario does and is in this way able to account for the outer planet in PSR $1257+12$. His results are therefore consistent with ours.

Planet formation will become possible if, later on, the disk of rocks can spread via collisions beyond the Roche limit. Given that collisions occur on relatively short, dynamical timescales, one

\footnotetext{
${ }^{15}$ Even if the disk survives the pulsar wind, it is possible for the wind to influence the disk evolution by providing an extra source of non-thermal ionization.
} 
would naively expect the disk to spread significantly over a system's lifetime, allowing late planet formation. As long as the disk lies inside the Roche limit, only small bodies, of the size of rocks or so, are allowed to form around the young neutron star in the proposed scenario. ${ }^{16}$ These objects are held together against tidal disruption by internal material strength. The tidal disruption radius under these conditions is (e.g. Aggarwal \& Oberbeck 1974)

$$
R_{\mathrm{tid}, \mathrm{int}} \approx\left(\frac{G M_{1} \rho r^{2}}{T}\right)^{1 / 3},
$$

where $r$ is the rock linear size and $\mathrm{T}$ its material strength. For example, an iron rock of material strength $T \sim 10^{10}$ dyne $\mathrm{cm}^{-2}$ (e.g. Mosenfelder et al. 2000) and size $r \sim 3 \times 10^{6} \mathrm{~cm}$ (roughly corresponding to a mass of $10^{21} \mathrm{~g}$ ) can exist only beyond $R_{\text {tid,int }} \sim 10^{10} \mathrm{~cm}$. Only smaller (less massive) rocks are allowed further in, with a distance-size relation obeying $R_{\text {tid,int }} \propto r^{2 / 3}$. For example, at a radius of $10^{8} \mathrm{~cm}$, only rocks with $r \lesssim 3 \times 10^{3} \mathrm{~cm}$ (mass $\lesssim 10^{12} \mathrm{~g}$ ) are allowed. The growth of rocks is limited by this maximum allowed mass spectrum. The collisional dynamics and global evolution of the disk are likely to be affected by the Roche-limited growth of its rocks. We defer a more detailed discussion of the evolution of such a disk of rocks to future work.

\subsection{The Black Hole Case}

All the results presented so far were calculated assuming a solar mass central object, i.e. a neutron star. We discuss here the extension of our work to the case of a central black hole. The results are qualitatively similar to the neutron star case. Assuming a black hole of mass $M_{1}=10 M_{\odot}$, the coefficients $\beta$ and $\beta_{\text {irr }}$ in the global stability criteria given in $\S 3$ become $\approx 1 / 8$ and $2 / 5$, respectively. The amount of fallback during a supernova explosion resulting in the formation of a black hole is larger than for a neutron star (see, e.g., Woosley \& Weaver 1995 for examples with several solar masses of fallback). The numerical simulations of the evolution of massive stars by Heger, Langer \& Woosley (2000) suggest that a specific angular momentum $j \sim 10^{17} \mathrm{~cm}^{2} \mathrm{~s}^{-1}$ for the fallback material is also a reasonable estimate for the black hole case. Below, we give specific results for a fallback disk around a black hole of mass $M_{1}=10 M_{\odot}$, a disk initial mass $M_{d}\left(t_{0}\right)=10^{-3} M_{\odot}$, a disk initial radius $R_{d}\left(t_{0}\right)=10^{7} \mathrm{~cm}$ (corresponding to the value of $j$ quoted above) and neglecting the factor of three difference in the initial viscous timescale (Eq. 8) for this more massive central object.

For these parameters, a non-irradiated disk becomes neutral when

$$
\begin{aligned}
t_{n} & \approx 6.3 \times 10^{3} \mathrm{yrs}\left(\times T_{c, 6}^{-21 / 37}\right), \\
R_{d}\left(t_{n}\right) & \approx 1.5 \times 10^{10} \mathrm{~cm}\left(\times T_{c, 6}^{6 / 37}\right)
\end{aligned}
$$

\footnotetext{
${ }^{16}$ In that respect, the system of rings around Saturn may offer some guidance as to the type of behavior to expect. The collisional dynamics in the deep potential well of a compact object may be quite different, however.
} 


$$
\begin{aligned}
& \dot{M}_{d}\left(t_{n}\right) \approx 3.2 \times 10^{16} \mathrm{~g} \mathrm{~s}^{-1}\left(\times T_{c, 6}^{18 / 37}\right) \\
& M_{d}\left(t_{n}\right) \approx 1.9 \times 10^{-5} \mathrm{M}_{\odot}\left(\times T_{c, 6}^{-3 / 37}\right) .
\end{aligned}
$$

The same disk, but weakly-irradiated, becomes neutral when

$$
\begin{aligned}
t_{n, w i} & \approx 1.2 \times 10^{5} \mathrm{yrs}\left(\times T_{c, 6}^{-15 / 31}\right), \\
R_{d}\left(t_{n, w i}\right) & \approx 4.5 \times 10^{10} \mathrm{~cm}\left(\times T_{c, 6}^{6 / 31}\right), \\
\dot{M}_{d}\left(t_{n, w i}\right) & \approx 8 \times 10^{15} \mathrm{~g} \mathrm{~s}^{-1}\left(\times T_{c, 6}^{12 / 31}\right), \\
M_{d}\left(t_{n, w i}\right) & \approx 1.5 \times 10^{-5} \mathrm{M}_{\odot}\left(\times T_{c, 6}^{-3 / 31}\right) .
\end{aligned}
$$

We do not consider the strongly-irradiated case: a disk around a black hole is even less subject to X-ray irradiation than a disk around a neutron star because there is no central X-ray source but only disk self-irradiation in this case (Shakura \& Sunyaev 1973). These results are somewhat different from the neutron star case, but have rather similar evolutionary implications.

Newly formed black holes surrounded by fallback disks should be active X-ray sources for relatively short periods of time. The immediate formation of planets is equally unlikely in the black hole case. Even for an extreme fallback disk mass of $1 M_{\odot}$, the maximal radius reached by the disk is $\sim 5 \times 10^{10} \mathrm{~cm}\left(2 \times 10^{11} \mathrm{~cm}\right)$ in the non-irradiated (weakly-irradiated) case. This is not larger than the tidal disruption radius $R_{\mathrm{tid}} \approx 3 \times 10^{11} \mathrm{~cm}$ for a self-gravitating body of density

$\rho \approx 1 \mathrm{~g} \mathrm{~cm}^{-3}$ in that case. Consequently, the outcome of the evolution of a fallback disk around a black hole is the formation of a disk of rocks, like in the neutron star case, with a maximum size (or mass) spectrum increasing with radius. Similarly, the formation of planets becomes possible only if the disk of rocks spreads beyond the Roche limit.

The identification of fallback disks around newly-formed black holes may prove extremely difficult once they become passive. We note, however, that the collisions experienced by the rocks in the disk will scatter some of them on orbits such that they will be accreted by the black hole. Rocks at $10^{11} \mathrm{~cm}$ with $r \lesssim 6 \times 10^{7} \mathrm{~cm}$ (mass $\lesssim 10^{25} \mathrm{~g}$; see Eq. 25]) would lead to events of fluence $\lesssim 10^{45}$ ergs, while rocks at $10^{8} \mathrm{~cm}\left(r \lesssim 2 \times 10^{3} \mathrm{~cm}\right.$; mass $\left.\lesssim 3 \times 10^{11} \mathrm{~g}\right)$ would lead to events of fluence only $\lesssim 3 \times 10^{31} \mathrm{ergs}$. Of course, the collisional dynamics of the disk of rocks could limit their growth and make them, on average, less massive than the maximal value allowed by tidal forces (assumed here for the fluence estimates). The duration of the accretion events will depend on the precise orbits of the rocks as they approach the black hole (see Colgate \& Petschek 1981 and Tremaine \& Zytkow 1986 for a discussion of comets accreting onto magnetized neutron stars).

\subsection{Doubly Degenerate Mergers}

The existence of a population of doubly degenerate (white dwarf - white dwarf) binaries in our galaxy has clearly been established, some of which are expected to merge within a Hubble 
time (e.g. Marsh 1995; Marsh, Dhillon \& Duck 1995). If the merger does not result in a Type Ia supernova (Whelan \& Iben 1973; Wheeler 1982; Webbink 1984), the end product is a massive white dwarf, or a neutron star if core-collapse occurs. Numerical simulations show that a large fraction of the angular momentum of the initial binary ends up in a disk surrounding the merger product. Whether this disk is composed primarily of helium or metals, it is subject to the thermal ionization instability $(\S 2)$ and presumably follows the same general evolutionary scenario as fallback disks $(\S 3)$. This is true independent of the nature of the merged compact object.

The typical mass and angular momentum in a disk resulting from the merger of two white dwarfs are significantly larger than those considered for fallback disks in $\S 3$. Benz et al. (1990; see also Segretain, Chabrier \& Mochkovitch 1997) find that the outer, rotationally-supported region of the merger product has a mass $\sim 0.3 M_{\odot}$, at radii $\sim 10^{9} \mathrm{~cm}$. Using these values for the initial disk mass and radius in the evolutionary model of $\S 3.1$ yields the following conditions for the disk when it becomes neutral:

$$
\begin{aligned}
t_{n} & \approx 1.8 \times 10^{3} \mathrm{yrs}\left(\times T_{c, 6}^{-21 / 37}\right), \\
R_{d}\left(t_{n}\right) & \approx 3.9 \times 10^{11} \mathrm{~cm}\left(\times T_{c, 6}^{6 / 37}\right), \\
\dot{M}_{d}\left(t_{n}\right) & \approx 6 \times 10^{20} \mathrm{~g} \mathrm{~s}^{-1}\left(\times T_{c, 6}^{18 / 37}\right), \\
M_{d}\left(t_{n}\right) & \approx 1.5 \times 10^{-2} \mathrm{M}_{\odot}\left(\times T_{c, 6}^{-3 / 37}\right) .
\end{aligned}
$$

Consequently, it appears possible for such a disk to spread enough before becoming neutral to allow planet formation after the activity period, in regions located beyond the Roche limit at $\sim 10^{11} \mathrm{~cm}$. This scenario suggests that massive white dwarfs which are the result of a doubly degenerate merger are capable of harboring planets (see Livio, Pringle \& Saffer [1992] for a similar conclusion but a different line of reasoning). Similarly, in case of core-collapse following the merger, the neutron star end product will also be a potential host for planets. We note, however, that a necessary ingredient for planet formation to occur in the doubly degenerate context is that the secondary in the pre-merger binary was massive enough to be mostly composed of Carbon/Oxygen rather than Helium.

\subsection{Other Applications}

In addition to the X-ray point sound discovered recently in Cas A with the Chandra satellite, Chakrabarty et al. (2000) discuss three radio-quiet, non-plerionic X-ray point sources which have been associated with supernova remnants. These X-ray sources could be explained by emission from a fallback accretion disk. As noted by Chakrabarty et al., the radio-quiet X-ray point sources share characteristics in common with AXPs. The absence of X-ray pulsations may indicate the presence of a black hole accretor in these systems. We note, however, that the X-ray luminosities of these four X-ray sources are typically one or two orders of magnitude below those typical of AXPs. This could be accounted for by the fallback scenario only for relatively small initial disk 
mass and angular momentum.

Fallback disks could also be present around newly-formed compact objects following core-collapse supernova explosions in binary systems. We note that, in this context, it is in principle possible for the fallback material to acquire some extra angular momentum relative to the compact object during the explosion because of the non-axisymmetric tidal perturbations induced by the companion. The presence of the disk will not substantially influence the orbital evolution of the binary because the angular momentum in the companion's orbit far exceeds that in the disk. Except perhaps in close binaries, the fallback disk may not spread enough to interact strongly with the companion before it becomes neutral (although the eccentric orbit of the companion, following the supernova explosion, would favor such an interaction). Independent of whether the disk is in the gaseous phase or made of rocks, the effect of the presence of the companion will be to remove angular momentum from the disk via tidal interactions (Goldreich \& Tremaine 1980).

\section{Discussion}

We presented in $\S 3$ simple estimates for the age, size and accretion rates at which supernova fallback disks are expected to become neutral under the action of the thermal ionization instability. We stress here that these estimates are only approximate because of the various simplifying assumptions made in our derivations. We do not know the exact composition of a fallback disk $\square$, so we ignore its precise stability properties. The strength of disk irradiation, which also influences the stability properties, is not well-determined either. We treated the viscous evolution of an irradiated disk only in a simplified manner. We used similarity solutions to describe the evolution of a disk of finite (and generally small) radial extent, while the solution applies best to the large extent case. Although all these approximations are sources of uncertainty, we find that, quite generally, the model predicts that fallback disks should become neutral when they are still young, compact and accreting at generally sub-Eddington rates.

Another important source of uncertainty for the models described in $\S 3$ comes from the early evolution of the fallback disk, when it accretes at super-Eddington rates. We did not differentiate this phase from the sub-Eddington one, but the relevance of the similarity solutions applied to the super-Eddington phase is not guaranteed. A variety of viscosity laws would predict a power-law evolution for the accretion rate in the disk with an index close to -1.2 as considered in $\S 3$ (Lynden-Bell \& Pringle 1974), so that this type of solution is rather robust. On the other hand, the assumption of accretion via a thin disk may not be valid during the super-Eddington phase. It is conceivable that the disk evolution will still proceed according to the power law solutions used in $\S 3$ if the disk finds ways to cool efficiently, e.g. because of the "photon bubble" instability

\footnotetext{
${ }^{17}$ The composition of the fallback material depends on the amount of mass that makes it in the disk. For a disk with small mass, say $M \leq 10^{-3} M_{\odot}$, the fallback material will likely be made of silicon-burning products.
} 
identified by Gammie (1998), but the evolution during the super-Eddington phase definitely constitutes an uncertainty for the models. If the disk were unable to cool, the initial viscous evolution would proceed much faster and the global outcome could be significantly affected. This uncertainty is essentially captured in the scaling with $T_{c, 6}$ in all our equations. A rather robust ingredient of the models appears to be the compact size of fallback disks when they become neutral. We show explicitly in Appendix B that fallback disks with arbitrarily fast power-law evolutions are still restricted to rather small sizes $(<1 \mathrm{AU})$.

The models described in $\S 3$ assume that the total angular momentum of the fallback disk is conserved during its evolution. It is possible for a central neutron star, however, to transfer a fraction of its spin angular momentum to the disk via magnetospheric interaction. The angular momentum of the neutron star is

$$
A_{\mathrm{NS}}=I \Omega \approx 4 \times 10^{47}\left(\frac{P}{15 \mathrm{~ms}}\right)^{-1} \mathrm{~g} \mathrm{~cm}^{2} \mathrm{~s}^{-1},
$$

where $I\left(\approx 10^{45} \mathrm{~g} \mathrm{~cm}^{2} \mathrm{~s}^{-1}\right)$ and $P$ are the moment of inertia and spin period of the neutron star, respectively. The total angular momentum of the disk is

$$
\begin{aligned}
A_{\mathrm{disk}} & \approx M_{d}\left(t_{0}\right)\left(G M_{1} R_{d}\left(t_{0}\right)\right)^{1 / 2} \\
& \approx 3 \times 10^{47}\left(\frac{M_{d}\left(t_{0}\right)}{10^{-3} \mathrm{M}_{\odot}}\right)\left(\frac{R_{d}\left(t_{0}\right)}{10^{8} \mathrm{~cm}}\right)^{1 / 2} \mathrm{~g} \mathrm{~cm}^{2} \mathrm{~s}^{-1}
\end{aligned}
$$

where $M_{1}=1.4 M_{\odot}$ is the mass of the central object. Clearly, in the case of a rapidly spinning neutron star and a low angular momentum disk, the contribution from the spun down neutron star to the disk angular momentum can become important or even dominant over time.

This possibility is not a problem in the magnetospheric interaction model of Chatterjee et al. (2000) because the initially fast spinning neutron star loses most of its angular momentum during an early propeller phase, so that most of the stellar angular momentum is lost to infinity. By the time the disk enters the "tracking phase" and acquires angular momentum by spinning down the neutron star, now having a period of several seconds, the stellar angular momentum of the neutron star is at most comparable to that of the disk except for a fallback disk with very small initial angular momentum.18 On the other hand, if this early propeller phase were not to occur, the viscous evolution of a fallback disk could be mainly determined by the angular momentum that it receives from the neutron star. In the limit where the disk mass is conserved and its evolution is driven by the torque applied at its inner edge by the neutron star, it would follow the power law solutions found by Pringle (1991) for "external disks".

The analogy between fallback disks and disks in close binaries made in $\S 3$ is also useful if, contrary to what we argued, fallback disks do not become passive when they become neutral. If fallback disks were to possess a residual viscosity in their neutral phase, with a magnitude

\footnotetext{
${ }^{18}$ The specific AXP disk model discussed in $\S 4.1 .1$ is safe in that respect.
} 
significantly smaller than that of the ionized phase (because, for instance, of a mechanism different from MHD turbulence operating in the neutral disk), numerical disk instability models suggest that the disks would then experience outbursts similar to those of transient close binaries. They would do so until the accretion rate in the disk is so small that the entire disk is neutral and no longer subject to the thermal ionization instability. In first approximation, one would expect the period of evolution with outbursts to last at least a time comparable to the disk lifetimes estimated in $\S 3$, although a more detailed numerical investigation is probably required to answer this question properly. The fact that no such bright, isolated soft X-ray transient has been discovered to date does not seem to support this scenario. On the other hand, if the magnitude of the viscosity in the neutral phase of fallback disks is comparable to that in the ionized phase (e.g. because large opacities leading to efficient MHD coupling), the disks would only experience very small amplitude luminosity fluctuations due to multiple reflections of recombination/ionization fronts (Smak 1984; Hameury et al. 1998; Menou et al. 1999). It may be reasonable to expect the disk evolution to still proceed in a self-similar manner in that case, until the central temperature at the disk outer edge reaches a temperature $(\sim 2000 \mathrm{~K})$ at which the gas and the magnetic field in the disk are no longer coupled. This evolutionary scenario cannot be further characterized without a better knowledge of the opacity in the neutral metal-rich disk, but we note that it may lead to a passive neutral disk in a final state not that different from those discussed in $\S 3$.

We emphasized in $\S 4.1$ an application of the evolutionary models for fallback disks developed in $\S 3$ to Anomalous X-ray Pulsars (AXPs). These sources, because of similarities, have been associated with Soft Gamma Repeaters (SGRs). There is no component in the fallback accretion disk scenario described here which can explain the burst phenomenology of SGRs. We also note that Thompson et al. (1999) have presented strong arguments against standard viscous accretion powering the quiescent emission of SGRs. It may be possible to explain the SGR phenomenology by considering the evolution of a fallback disk after it turns to rocks, but the situation is presently unclear. Along those lines, we note that Katz, Toole \& Unruh (1994) have proposed an SGR model in which planetary collisions and subsequent accretion onto a central neutron star power SGR bursts. We suspect that this scenario is unlikely to apply to the disk of rocks formed after fallback disk evolution because of the large mass objects required to power some of the most luminous SGR bursts observed and the absence of eccentric orbits favoring large body collisions in the disk (the eccentricities in the planetary disk predating the supernova explosion in the scenario described by Katz et al. 1994 are caused by the explosion itself).

\section{Conclusions}

We have shown that thin accretion disks composed of Carbon, Oxygen and probably any other metal are subject to the same thermal ionization instability as Hydrogen and Helium disks. While the instability is thought to be responsible for large-amplitude outbursts in dwarf novae and X-ray transients, we argued that its role could be different for supernova fallback disks. As 
the instability quickly turns such a metal-rich disk into a neutral medium, there may not be any viscosity source left in the unmagnetized disk that is sufficient to prevent dust formation. The outcome of the instability in this context would therefore be a passive disk of dust which would later evolve into a disk of rocks via coagulation processes.

We constructed simple analytical models to predict the time at which a supernova fallback disk becomes neutral. For reasonable values of the disk initial mass and angular momentum, we find that this happens when the disks are still young, compact in size and generally accreting at sub-Eddington rates. A direct application of the models shows that they can account for disks of age a few $10^{3} \mathrm{yrs}$, radial extent of a few $10^{9} \mathrm{~cm}$ and accretion rate $\sim 10^{16} \mathrm{~g} \mathrm{~s}^{-1}$, as inferred for hypothetical disks in Anomalous X-ray Pulsars.

The late evolution of the disk of rocks formed around a young compact object will strongly depend on its ability to spread via collisions. The dynamics of this disk may be heavily influenced by the fact that it generally lies inside the tidal disruption radius for self-gravitating bodies (Roche limit), at $\approx 10^{11} \mathrm{~cm}$. The formation of planets around the compact object will become possible only if/when the disk spreads beyond this Roche limit.

\section{Acknowledgments}

We thank Guillaume Dubus, Jeremy Goodman, Brad Hansen, Ramesh Narayan, Bohdan Paczynski, Eliot Quataert and Scott Tremaine for very useful discussions. Support for this work

was provided by NASA through Chandra Fellowship grant PF9-10006 awarded by the Smithsonian Astrophysical Observatory for NASA under contract NAS8-39073.

\section{REFERENCES}

Aggarwal, H.R. \& Oberbeck, V.R. 1974, ApJ, 191, 577

Alexander, D.R. \& Ferguson, J.W. 1994, ApJ, 437, 879

Alpar, M.A. 1999, preprint, astro-ph/9912228

Alpar, M.A. 2000, ApJ, submitted, astro-ph/0005211

Balbus, S. A., \& Hawley, J.F. 1991, ApJ, 376, 214

Balbus, S. A., \& Hawley, J.F. 1998, Rev. Mod. Phys., 70, 1

Balbus, S. A. \& Terquem, C. 2001, ApJ, submitted, astro-ph/0010229

Benz, W., Bowers, R.L., Cameron, A.G.W. \& Press, W.H. 1990, ApJ, 348, 647

Cabot, W. 1996, ApJ, 465, 874 
Cannizzo, J.K. 1984, Nature, 311, 443

Cannizzo, J.K. 1993a, ApJ, 419, 318

Cannizzo, J.K. 1993b, in Wheeler J.C., ed., Accretion discs in Compact Stellar Systems (World Scientific, Singapore), p. 6

Cannizzo, J.K., Lee, H.M. \& Goodman, J. 1990, ApJ, 351, 38

Chakrabarty, D., Pivovaroff, M.J., Hernquist, L.E., Heyl, J.S. \& Narayan, R., 2001, ApJ, in press, astro-ph/0001026

Chatterjee, P. \& Hernquist, L. 2000, ApJ, 543, 368

Chatterjee, P., Hernquist, L. \& Narayan, R. 2000, ApJ 534, 373

Chevalier, R.A. 1989, ApJ, 346, 847

Colgate, S.A. 1988, in Supernova 1987A in the Large Magellanic Cloud; Proceedings of the Fourth George Mason Astrophysics Workshop, Fairfax, p. 341

Colgate, S.A. \& Petschek, A.G. 1981, ApJ, 248, 771

Cunningham, C. 1976, ApJ, 208, 534

Dubus, G., Hameury, J.-M. \& Lasota, J.-P., 2001, preprint

Dubus, G., Lasota, J.-P., Hameury, J.-M. \& Charles, P. 1999, MNRAS, 303, 139

El-Khoury, W. \& Wickramasinghe, D. 2000, A\&A, 358, 154

Fleming, T.P., Stone, J.M. \& Hawley, J.F., 2000, ApJ, 530, 464

Frank, J., King, A. \& Raine, D., 1992, Accretion Power in Astrophysics (Cambridge Univ. Press, Cambridge)

Fryer, C.L. \& Heger, A. 2000, 541, 1033

Gammie, C.F. 1996, ApJ, 457, 355

Gammie, C.F. 1998, MNRAS, 297, 929

Gammie C.F. \& Menou K., 1998, ApJ, 492, L75

George, I.M. \& Fabian, A.C. 1991, MNRAS, 249, 352

Goldreich, P. \& Tremaine, S. 1980, ApJ, 241, 425

Goodman, J. 1993, ApJ, 406, 596 
Hameury J.-M., Menou K., Dubus G., Lasota J.-P., Huré J.-M., 1998, MNRAS, 298, 1048

Hansen, B.M.S. 2001, in Stellar Collisions, Mergers and their Consequences, ASP Conference Series, ed. M. Shara, astro-ph/0008226

Hawley, J.F., Balbus, S.A. \& Winters, W.F. 1999, ApJ, 518, 394

Hawley, J. F., Gammie, C. F., \& Balbus, S. 1996, ApJ, 464, 690

Heger, A., Langer, N. \& Woosley, S.E. 2000, ApJ, 528, 368

Heyl, J.S. \& Hernquist, L. 1997, ApJ, 489, L67

Hulleman, F., van Kerkwijk, M.H. \& Kulkarni, S.R. 2000b, Nature, 408, 689

Hulleman, F., van Kerkwijk, M.H., Verbunt, F.W.M. \& Kulkarni, S.R. 2000a, A\&A, 358, 605

Iglesias, C.A. \& Rogers, F.J. 1996, ApJ, 464, 943

Illarionov, A.F. \& Sunyaev, R.A. 1975, A\&A, 39, 185

Katz, J.I., Toole, H.A. \& Unruh, S.H. 1994, ApJ, 437, 727

Lasota, J.-P. 2001, New Astron. Rev., in press, astro-ph/0102072

Lin, D.N.C., Woosley, S.E. \& Bodenheimer, P.H. 1991, Nature, 353, 827

Livio, M., Pringle, J.E. \& Saffer, R.A. 1992, MNRAS, 257, 15

Ludwig, K., Meyer-Hofmeister, E. \& Ritter, H. 1994, A\&A, 290, 473

Lynden-Bell, D. \& Pringle, J.E., 1974, MNRAS, 168, 603

Marsden, D., Lingenfelter, R.E. \& Rothschild, R.E. 2001a, ApJL, in press, astro-ph/0008300

Marsden, D., Lingenfelter, R.E. \& Rothschild, R.E. 2001b, preprint, astro-ph/0102049

Marsh, T.R., 1995, MNRAS, 275, L1

Marsh, T.R., Dhillon, V.S. \& Duck, S.R., 1995, MNRAS, 275,828

Menou, K., 2000, Science, 288, 2022

Menou K., Hameury J.-M., Stehle R., 1999, MNRAS, 305, 79

Menou, K. \& Quataert, E. 2001, ApJ, in press, astro-ph/0008368

Meyer F., Meyer-Hofmeister E., 1981, A\&A, 104, L10

Meyer-Hofmeister E., 1992, A\&A, 253, 459 
Michel, F.C. 1988, Nature, 333, 644

Michel, F.C. \& Dessler, A.J. 1981, ApJ, 251, 654

Michel, F.C. \& Dessler, A.J. 1983, Nature, 303, 48

Mihalas, D., Hummer, D.G., Mihalas, B.W. \& Dappen, W. 1990, ApJ, 350,300

Mineshige, S., Nomoto, K. \& Shigeyama, T., 1993, A\&A, 267, 95

Mosenfelder, J.L., Connolly, J.A.D., Rubie, D.C. \& Liu, M. 2000, Phys. Earth Plan. Interiors, 120, 63

Narayan, R., Mahadevan, R. \& Quataert, E., 1998b, in The Theory of Black Hole Accretion Discs, eds. M. A. Abramowicz, G. Bjornsson, and J. E. Pringle (Cambridge: Cambridge University Press), astro-ph/9803141.

Osaki, Y. 1996, PASP, 108, 39

Perna, R., Hernquist, L., \& Narayan, R. 2000, ApJ, 541, 344

Perna, R. \& Hernquist, L. 2000, ApJ, 544, L57

Phinney, E.S. \& Hansen, B.M.S. 1993, in Planets around pulsars, Proceedings of the Conference, Caltech, Pasadena, p. 371-390.

Pringle, J.E. 1974, Ph.D. Thesis, University of Cambridge

Pringle, J.E. 1991, MNRAS, 248, 754

Richard, D. \& Zahn, J.-P. 1999, A\&A, 347, 734

Ryu, D. \& Goodman, J. 1992, ApJ, 388, 438

Segretain, L., Chabrier, G. \& Mochkovitch, R. 1997, ApJ, 481, 355

Shakura, N.I. \& Sunyaev, R.A. 1973, A\&A, 24, 337

Smak J., 1983, Acta Astron., 33, 333

Smak J., 1984, Acta Astron., 34, 161

Spruit, H C. 1987, A\&A, 184, 173

Stone, J.M. \& Balbus, S.A. 1996, ApJ, 464, 364

Thompson, C. \& Duncan, R.C. 1996, ApJ, 473, 322

Thompson, C. et al. 2000, ApJ, 543, 340 
Tremaine, S. \& Zytkow, A.N. 1986, ApJ, 301, 155

Tsugawa, M. \& Osaki, Y. 1997, PASJ 49, 75

van Paradijs, J. 1996, ApJL, 464, L139

Vrtilek, S.D., Raymond, J.C., Garcia, M.R., Verbunt, F., Hasinger, G. \& Kurster, M. 1990, A\&A, 235,162

Warner, B. 1995, Cataclysmic variable stars (Cambridge University Press, Cambridge)

Warner, B., Nather, R. E., \& MacFarlane, M. 1969, Nature, 222, 233

Webbink, R.F., 1984, ApJ, 277, 355

Wheeler, J.C., 1982, in Supernovae: A survey of current research, eds. M.J. Rees \& R.J. Stoneham (Dordrecht: Reidel), p. 167

Whelan, J. \& Iben, I.Jr., 1973, ApJ, 186, 1007

Wolszczan, A. 1994, Science, 264, 538

Wolszczan, A. \& Frail, D.A. 1992, Nature, 355, 145

Woosley, S.E. \& Weaver, T.A. 1995, ApJS, 101, 181 


\section{A. Power-Law Evolution of Irradiated Fallback Disks}

In this Appendix, we derive analytical solutions for the evolution of a supernova fallback disk, under two different assumptions for the strength of irradiation.

\section{A.1. Weakly-Irradiated Disk}

In this case, we assume that the disk evolution proceeds as for a non-irradiated $\alpha$-disk, but the stability criterion including the heating by irradiation is used (Eq. 20]). A derivation similar

to that presented in $\S 3.1$ shows that the outermost disk annulus becomes neutral in that case after a time:

$$
t_{n, w i} \approx 1.2 \times 10^{4} \mathrm{yrs}\left(\frac{M_{d}\left(t_{0}\right)}{\beta_{i r r} 10^{-3} \mathrm{M}_{\odot}}\right)^{16 / 31} T_{c, 6}^{-15 / 31} R_{d, 8}^{-49 / 62}\left(t_{0}\right) .
$$

This happens for a disk outer radius

$$
R_{d, 8}\left(t_{n, w i}\right) \approx 1.25 \times 10^{3}\left(\frac{M_{d}\left(t_{0}\right)}{\beta_{i r r} 10^{-3} \mathrm{M}_{\odot}}\right)^{6 / 31} T_{c, 6}^{6 / 31} R_{d, 8}^{16 / 31}\left(t_{0}\right)
$$

a disk mass

$$
M_{d}\left(t_{n, w i}\right) \approx 2.8 \times 10^{-5} \mathrm{M}_{\odot} \beta_{i r r}^{3 / 31}\left(\frac{M_{d}\left(t_{0}\right)}{10^{-3} \mathrm{M}_{\odot}}\right)^{28 / 31} T_{c, 6}^{-3 / 31} R_{d, 8}^{15 / 62}\left(t_{0}\right),
$$

and a disk accretion rate

$$
\dot{M}_{d}\left(t_{n, w i}\right) \approx 1.5 \times 10^{17} \mathrm{~g} \mathrm{~s}^{-1} \beta_{i r r}^{19 / 31}\left(\frac{M_{d}\left(t_{0}\right)}{10^{-3} \mathrm{M}_{\odot}}\right)^{12 / 31} T_{c, 6}^{12 / 31} R_{d, 8}^{32 / 31}\left(t_{0}\right) .
$$

For a low mass and low angular momentum disk $\left(M_{d}\left(t_{0}\right)=10^{-6} \mathrm{M}_{\odot}, R_{d, 8}\left(t_{0}\right)=0.01\right.$ and $\beta_{\text {irr }}=1$ ), this gives:

$$
\begin{aligned}
t_{n, w i} & \approx 1.3 \times 10^{4} \mathrm{yrs}\left(\times T_{c, 6}^{-15 / 31}\right) \\
R_{d}\left(t_{n, w i}\right) & \approx 3 \times 10^{9} \mathrm{~cm}\left(\times T_{c, 6}^{6 / 31}\right) \\
\dot{M}_{d}\left(t_{n, w i}\right) & \approx 10^{14} \mathrm{~g} \mathrm{~s}^{-1}\left(\times T_{c, 6}^{12 / 31}\right)
\end{aligned}
$$

For a high mass and high angular momentum disk $\left(M_{d}\left(t_{0}\right)=10^{-2} \mathrm{M}_{\odot}, R_{d, 8}\left(t_{0}\right)=1\right.$ and $\beta_{\text {irr }}=1$ ), this gives:

$$
\begin{aligned}
t_{n, w i} & \approx 4 \times 10^{4} \mathrm{yrs}\left(\times T_{c, 6}^{-15 / 31}\right), \\
R_{d}\left(t_{n, w i}\right) & \approx 2 \times 10^{11} \mathrm{~cm}\left(\times T_{c, 6}^{6 / 31}\right), \\
\dot{M}_{d}\left(t_{n, w i}\right) & \approx 3.6 \times 10^{17} \mathrm{~g} \mathrm{~s}^{-1}\left(\times T_{c, 6}^{12 / 31}\right) .
\end{aligned}
$$

As before, it can easily be checked that the disk is still strongly optically thick to X-rays and its own radiation when it becomes neutral. 


\section{A.2. Strongly-Irradiated Disk}

We assume now that when the outer radius of the fallback disk reaches $10^{9} \mathrm{~cm}$, it switches to the more rapid evolution of a vertically isothermal disk (with a constant irradiation flux to

guarantee that the viscosity is time-independent). Before that, irradiation does not affect the disk evolution, which proceeds according to Eqs. (35). The properties of the fallback disk when it reaches $10^{9} \mathrm{~cm}$ are therefore given by:

$$
\begin{aligned}
t_{9} & =3.1 \times 10^{-2} \mathrm{yrs}\left[T_{c, 6}^{-1} R_{d, 8}^{-13 / 6}\left(t_{0}\right)\right] \\
M_{d}\left(t_{9}\right) & =0.32 M_{d}\left(t_{0}\right)\left[R_{d, 8}^{1 / 2}\left(t_{0}\right)\right] \\
\dot{M}_{d}\left(t_{9}\right) & =6.8 \times 10^{23} \mathrm{~g} \mathrm{~s}^{-1}\left[\left(\frac{M_{d}\left(t_{0}\right)}{10^{-3} \mathrm{M}_{\odot}}\right) T_{c, 6} R_{d, 8}^{8 / 3}\left(t_{0}\right)\right] .
\end{aligned}
$$

The subsequent viscous evolution of the disk, now assumed to be vertically isothermal and steadily irradiated, follows:

$$
\begin{aligned}
& M_{d}(t)=M_{d}\left(t_{9}\right)\left(\frac{t}{t_{9}}\right)^{-7 / 13}, \\
& R_{d}(t)=R_{d}\left(t_{9}\right)\left(\frac{t}{t_{9}}\right)^{14 / 13}, \\
& \dot{M}_{d}(t)=\dot{M}_{d}\left(t_{9}\right)\left(\frac{t}{t_{9}}\right)^{-20 / 13} .
\end{aligned}
$$

Using the global stability criterion that includes the heating by irradiation (Eq. [20]), we find that the fallback disk becomes neutral after a time:

$$
t_{n, s i} \approx 26.7 \mathrm{yrs}\left(\frac{M_{d}\left(t_{0}\right)}{\beta_{i r r} 10^{-3} \mathrm{M}_{\odot}}\right)^{13 / 48} T_{c, 6}^{-35 / 48} R_{d, 8}^{-13 / 9}\left(t_{0}\right) .
$$

This happens for a disk outer radius

$$
R_{d, 8}\left(t_{n, s i}\right) \approx 1.4 \times 10^{4}\left(\frac{M_{d}\left(t_{0}\right)}{\beta_{i r r} 10^{-3} \mathrm{M}_{\odot}}\right)^{7 / 24} T_{c, 6}^{7 / 24} R_{d, 8}^{7 / 9}\left(t_{0}\right),
$$

a disk mass

$$
M_{d}\left(t_{n, s i}\right) \approx 8.4 \times 10^{-6} \mathrm{M}_{\odot} \beta_{i r r}^{7 / 48}\left(\frac{M_{d}\left(t_{0}\right)}{10^{-3} \mathrm{M}_{\odot}}\right)^{41 / 48} T_{c, 6}^{-7 / 48} R_{d, 8}^{1 / 9}\left(t_{0}\right)
$$

and a disk accretion rate

$$
\dot{M}_{d}\left(t_{n, s i}\right) \approx 2 \times 10^{19} \mathrm{~g} \mathrm{~s}^{-1} \beta_{i r r}^{5 / 12}\left(\frac{M_{d}\left(t_{0}\right)}{10^{-3} \mathrm{M}_{\odot}}\right)^{7 / 12} T_{c, 6}^{7 / 12} R_{d, 8}^{14 / 9}\left(t_{0}\right) .
$$


For a low mass and low angular momentum disk $\left(M_{d}\left(t_{0}\right)=10^{-6} \mathrm{M}_{\odot}, R_{d, 8}\left(t_{0}\right)=0.01\right.$ and $\beta_{\text {irr }}=1$ ), this gives:

$$
\begin{aligned}
t_{n, s i} & \approx 3.2 \times 10^{3} \mathrm{yrs}\left(\times T_{c, 6}^{-35 / 48}\right) \\
R_{d}\left(t_{n, s i}\right) & \approx 5.2 \times 10^{9} \mathrm{~cm}\left(\times T_{c, 6}^{7 / 24}\right) \\
\dot{M}_{d}\left(t_{n, s i}\right) & \approx 2.7 \times 10^{14} \mathrm{~g} \mathrm{~s}^{-1}\left(\times T_{c, 6}^{7 / 12}\right)
\end{aligned}
$$

For a high mass and high angular momentum disk $\left(M_{d}\left(t_{0}\right)=10^{-2} \mathrm{M}_{\odot}, R_{d, 8}\left(t_{0}\right)=1\right.$ and $\beta_{\text {irr }}=1$ ), this gives:

$$
\begin{aligned}
t_{n, s i} & \approx 50 \mathrm{yrs}\left(\times T_{c, 6}^{-35 / 48}\right) \\
R_{d}\left(t_{n, s i}\right) & \approx 2.7 \times 10^{12} \mathrm{~cm}\left(\times T_{c, 6}^{7 / 24}\right) \\
\dot{M}_{d}\left(t_{n, s i}\right) & \approx 7.7 \times 10^{19} \mathrm{~g} \mathrm{~s}^{-1}\left(\times T_{c, 6}^{7 / 12}\right)
\end{aligned}
$$

Again, the disk is strongly optically thick to X-rays and its own radiation when this happens. 


\section{B. Disk Radial Extent for an Arbitrarily Fast Power-Law Evolution}

In this Appendix, we show that the radial extent of a fallback disk, when it becomes neutral, is finite and rather small even for an arbitrarily fast power law evolution in time. The derivation is made using the stability criterion for a non-irradiated disk (Eq. [2]) but a comparable result would be obtained using the criterion for an irradiated disk.

We parameterize the disk viscous evolution as follows:

$$
\begin{aligned}
M_{d}(t) & =M_{d}\left(t_{0}\right)\left(\frac{t}{t_{o}}\right)^{-p} \\
R_{d}(t) & =R_{d}\left(t_{0}\right)\left(\frac{t}{t_{0}}\right)^{2 p} \\
\dot{M}_{d}(t) & =\dot{M}_{d}\left(t_{0}\right)\left(\frac{t}{t_{0}}\right)^{-(1+p)},
\end{aligned}
$$

where $p>0$ for convergence. The time $t_{n}$ at which the disk becomes neutral is given by (Eq. [7]):

$$
\frac{t_{n}}{t_{0}} \approx\left(R_{d, 10}^{3}\left(t_{0}\right) \frac{\beta 10^{16} \mathrm{~g} \mathrm{~s}^{-1}}{\dot{M}_{d}\left(t_{0}\right)}\right)^{\frac{-1}{1+7 p}}
$$

The radius at which the outer disk becomes neutral is

$$
R_{d}\left(t_{n}\right) \approx R_{d}\left(t_{0}\right)\left(R_{d, 10}^{3}\left(t_{0}\right) \frac{\beta 10^{16} \mathrm{~g} \mathrm{~s}^{-1}}{\dot{M}_{d}\left(t_{0}\right)}\right)^{\frac{-2 p}{1+7 p}}
$$

with an asymptotic power law index of $-2 / 7$ at large $p$. In this limit, the corresponding value for a low mass and angular momentum disk $\left(M_{d}\left(t_{0}\right)=10^{-6} \mathrm{M}_{\odot}, R_{d, 8}\left(t_{0}\right)=0.01\right)$ is $R_{d}\left(t_{n}\right) \approx 5 \times 10^{9} \mathrm{~cm}$, while it yields $R_{d}\left(t_{n}\right) \approx 10^{12} \mathrm{~cm}$ for a disk with high mass and angular momentum $\left(M_{d}\left(t_{0}\right)=10^{-2} \mathrm{M}_{\odot}, R_{d, 8}\left(t_{0}\right)=1\right)$. This shows that disks with power-law evolutions faster than assumed in $\S 3$ are still restricted to sub-AU sizes. 


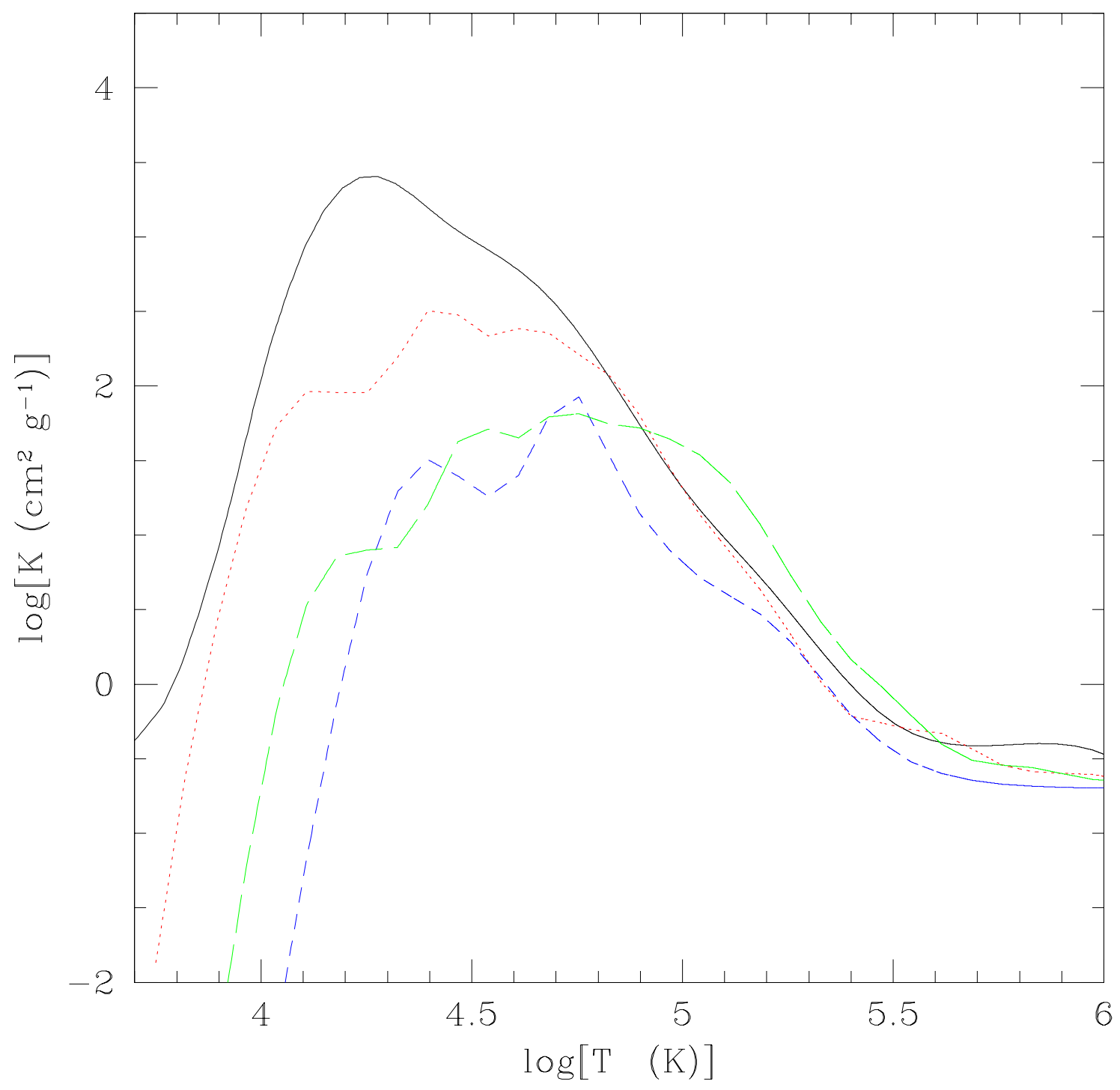

Fig. 1. - Rosseland-mean opacities as a function of temperature, at a mass density of $10^{-6} \mathrm{~g} \mathrm{~cm}^{-3}$, for solar composition material (solid line), pure Helium (short-dashed), pure Carbon (dotted) and pure Oxygen (long-dashed). In each case, the sudden opacity drop at $T \lesssim 10^{4} \mathrm{~K}$ corresponds to the recombination of the last available free electron. 

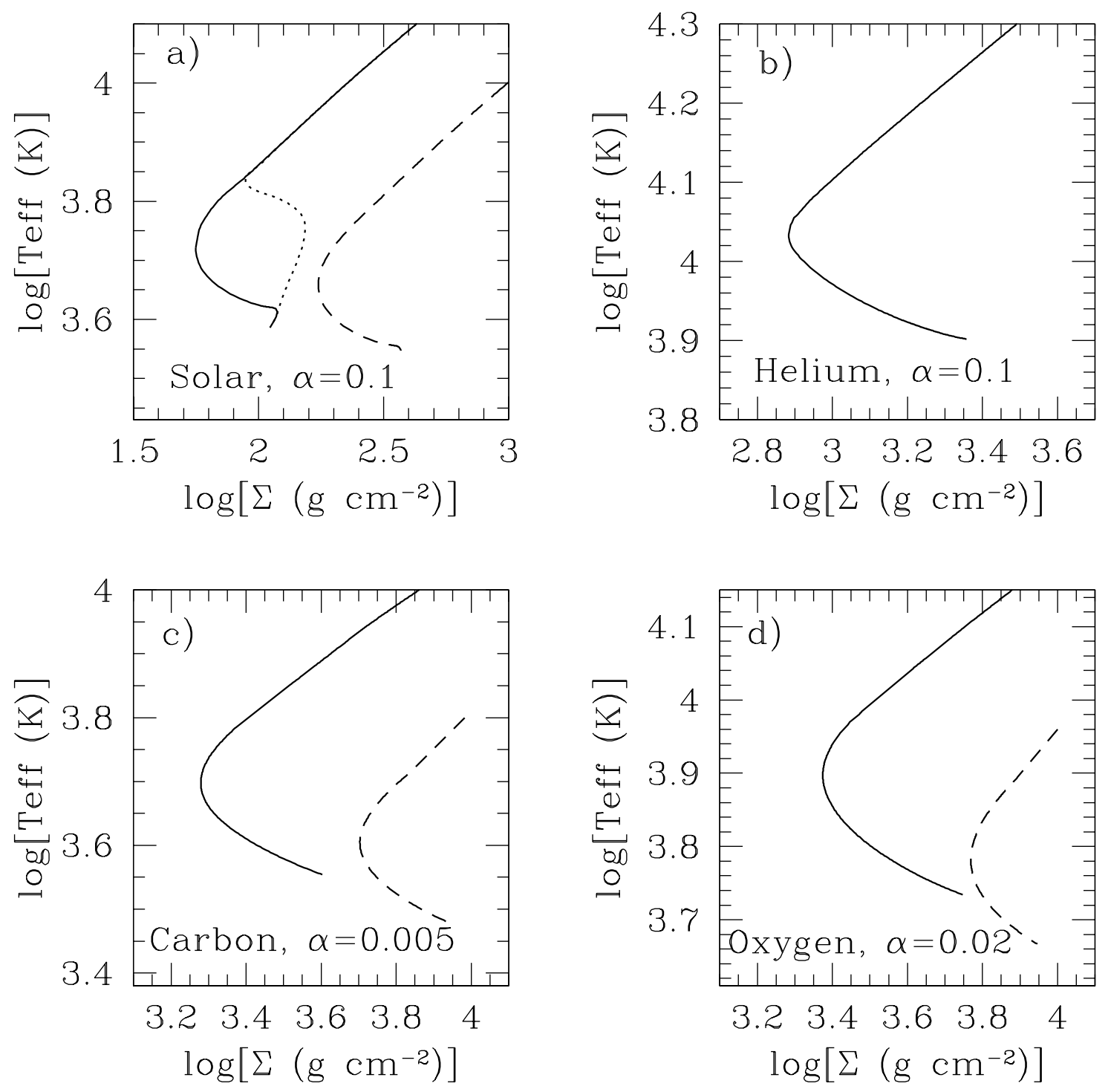

Fig. 2.- Examples of thermal equilibrium curves ("S-curves") for a thin accretion disk, shown in a surface density vs. effective temperature $\left(\Sigma-T_{\text {eff }}\right)$ diagram. The curves correspond to a disk annulus located at $2 \times 10^{10} \mathrm{~cm}$ (solid line) or $6 \times 10^{10} \mathrm{~cm}$ (dashed line) from a central neutron star of mass $M_{1}=1.4 M_{\odot}$. The four panels correspond to disks made of solar composition material (a), pure Helium (b), pure Carbon (c) and pure Oxygen (d). The value adopted for the viscosity parameter $\alpha$ is indicated in each panel. In panel (a), the dotted line shows the effect of allowing for convection in the disk vertical structure. In each case, the regions of the curves which have negative slopes indicate a thermally and viscously unstable disk (see text for details). 